Supplement of Atmos. Chem. Phys., 14, 10731-10740, 2014

http://www.atmos-chem-phys.net/14/10731/2014/

doi:10.5194/acp-14-10731-2014-supplement

(C) Author(s) 2014. CC Attribution 3.0 License.

(c) (1)

Supplement of

\title{
Climate-relevant physical properties of molecular constituents for isoprene-derived secondary organic aerosol material
}

M. A. Upshur et al.

Correspondence to: V. F. McNeill (vfm2103@columbia.edu), R. J. Thomson (r-thomson@northwestern.edu), and F. M. Geiger (geigerf@chem.northwestern.edu) 


\section{GAMMA 1.4 predictions of unreacted in-particle IEPOX concentrations.}

GAMMA 1.4 is based on GAMMA, the Gas-Aerosol Model for Mechanism Analysis. The full details of GAMMA 1.0 can be found in McNeill et al. (2012). GAMMA 1.4 includes the following updates:

1) Rate constant update for the aqueous reaction of the glyoxal radical with $\mathrm{O}_{2}: k^{I}=$ $1.2 \mathrm{e} 9\left[\mathrm{O}_{2}\right] 1 / \mathrm{s}$ based on Herrmann (2014)

2) Henry's Law constant updates: $\mathrm{H}^{*}=3 \mathrm{e} 7 \mathrm{M} / \mathrm{atm}$ for IEPOX (Nguyen et al., 2014) and $\mathrm{H}^{*}=2.7 \mathrm{e} 7 \mathrm{M} / \mathrm{atm}$ for glyoxal based on chamber data analysis of (Sumner, Woo, \& McNeill, 2014)

3) Added in-particle IEPOX reaction with $\mathrm{NH}_{4}{ }^{+}$, using a rate of $1.7 \mathrm{e}-51 / \mathrm{s}^{*}\left[\mathrm{NH}_{4}{ }^{+}\right]$ based on our analysis of the laboratory data of Nguyen et al. (2014).

4) Added the gas phase IEPOXOO + NO reaction based on Xie et al. (2013)

5) Added methacrylic acid epoxide (MAE) chemistry following Lin et al. (2013)

We calculated the unreacted in-particle concentration of IEPOX for ammonium sulfate aerosols after a $12 \mathrm{~h}$ sunlit period in a low-NOx environment for a range of aerosol $\mathrm{pH}$ and RH conditions (Figure S1). Details of the test conditions (initial conditions, emissions, and deposition rates), can be found in the Supporting Information of McNeill et al. (2012). The Whitby (1978) size distribution was used for the aerosols, and aerosol liquid water content was calculated as a function of $\mathrm{RH}$ and aerosol $\mathrm{pH}$ using the Extended AIM Aerosol Thermodynamics Model, E-AIM (http://www.aim.env.uea.ac.uk/aim/aim.php) and an assumed $4.0 \mathrm{ug} \mathrm{m}^{-3}$ of sulfate mass (Tanner et al., 2009)

The in-particle reactions of IEPOX are acid-catalyzed, so the remaining unreacted IEPOX increases with increasing $\mathrm{pH}$. The IEPOX ring opening reactions are most 
efficient at $45 \% \mathrm{RH}$ (highly concentrated conditions) and $\mathrm{pH}=1$. Unreacted IEPOX concentrations range from 2.1 to $22.5 \mathrm{mM}$.

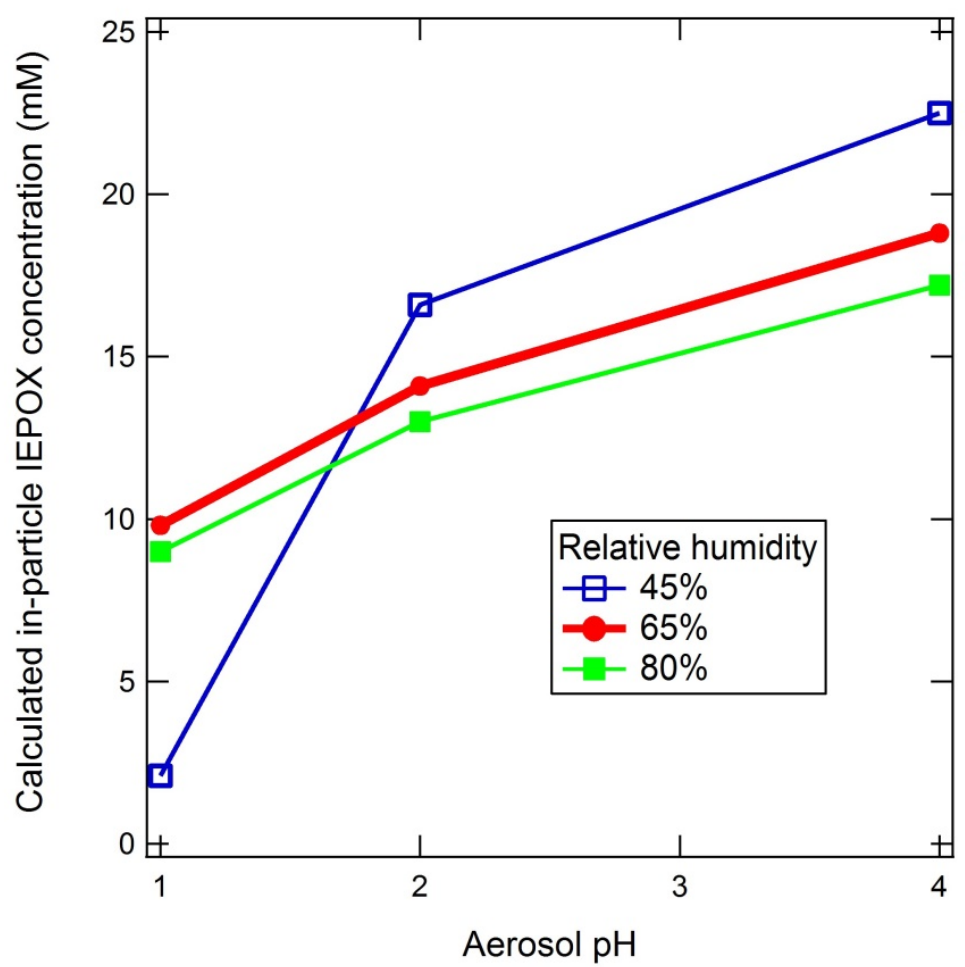

Figure S1. GAMMA 1.4 predictions of in -particle concentrations of unreacted IEPOX after $12 \mathrm{~h}$ simulation under remote conditions.

Figs. S2 and S3 show the GCMS traces and fragmentation patterns for the epoxides and tetraols studied in this work. Figs. S4-S6 show individual traces as well as overlaps of octanol chromatogram with chromatograms of selected IEPOX compounds and methyl vinyl ketone in octanol. 

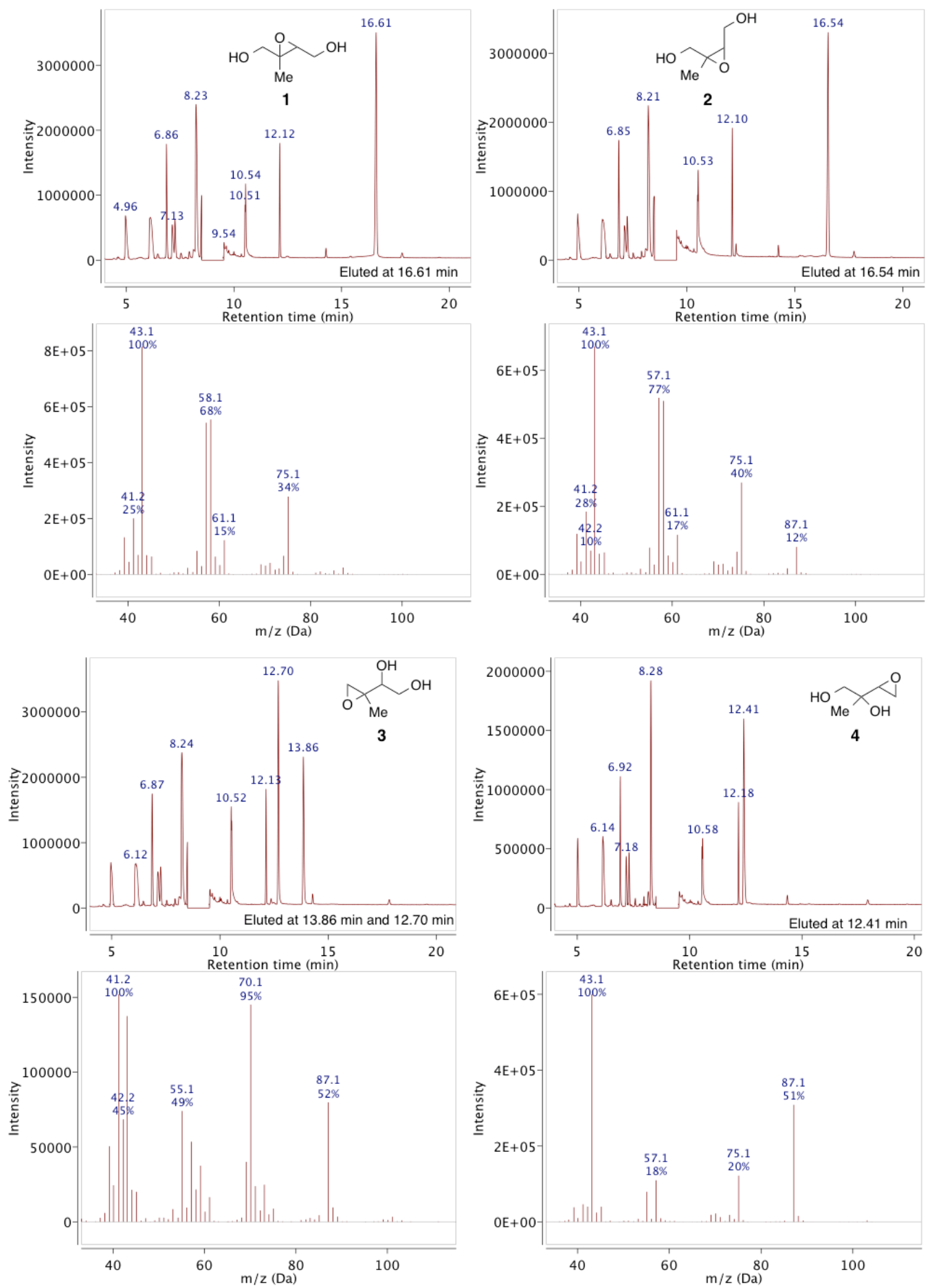

Figure S2. GC traces and fragmentation for IEPOX compounds (1-4). 

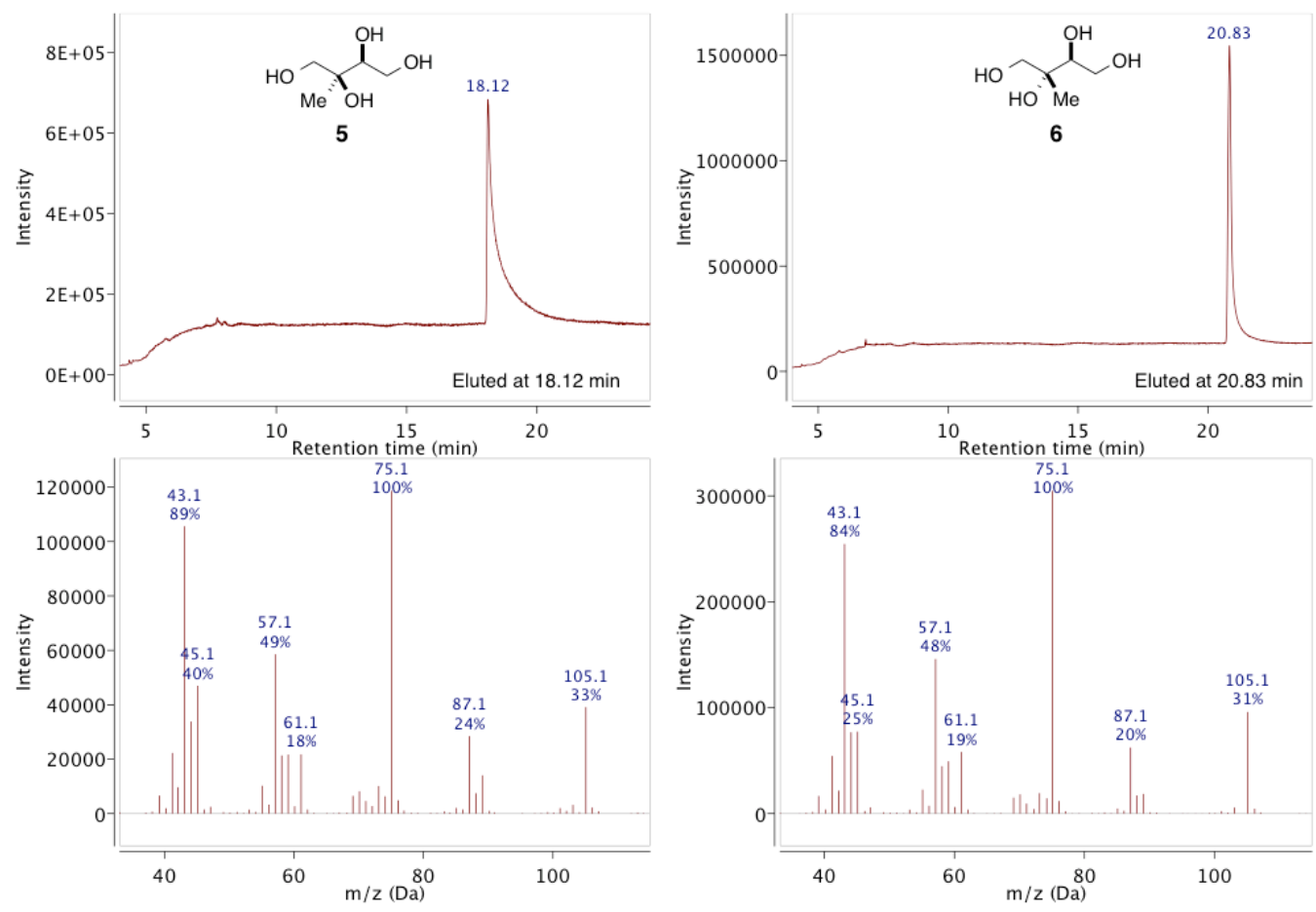

Figure S3. GC traces and fragmentation for 2-methyltetraol compounds $(5,6)$. Stock solutions of $\sim 40 \mathrm{mM}$ 2-methyltetraol in $\mathrm{dH}_{2} \mathrm{O}$ were used to obtain $\mathrm{GC}$ fragmentation due to limited solubility of 2-methyltetraol compounds in octanol. 

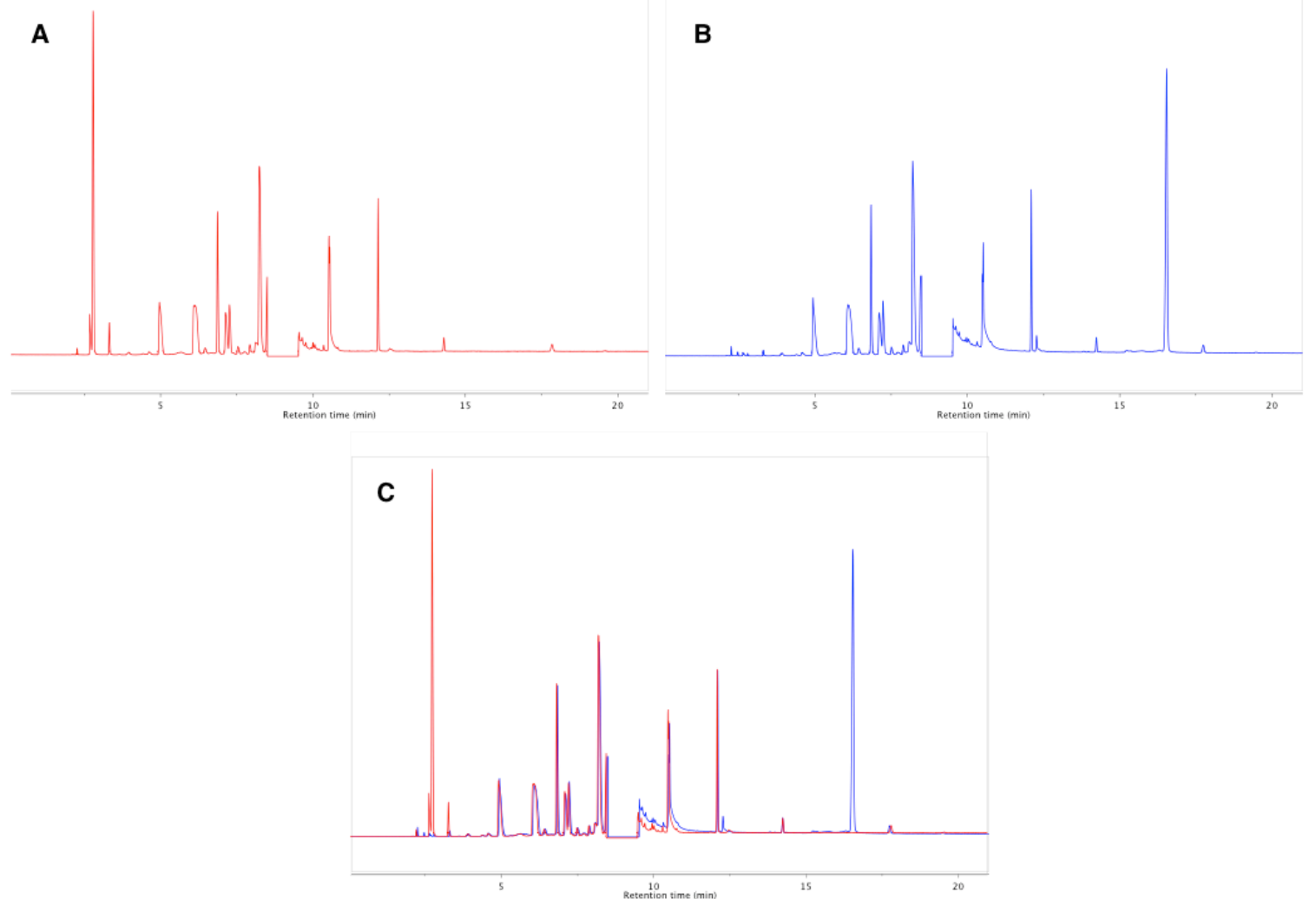

Figure S4. A) GC trace of pure octanol (red). B) GC trace of $45 \mathrm{mM}$ cis- $\beta$-IEPOX (2) dissolved in octanol (blue). C) Overlay of octanol trace (red) with cis- $\beta$-IEPOX (2)/octanol trace (blue). $C i s$ - $\beta$-IEPOX eluted at 16.54 minutes. 

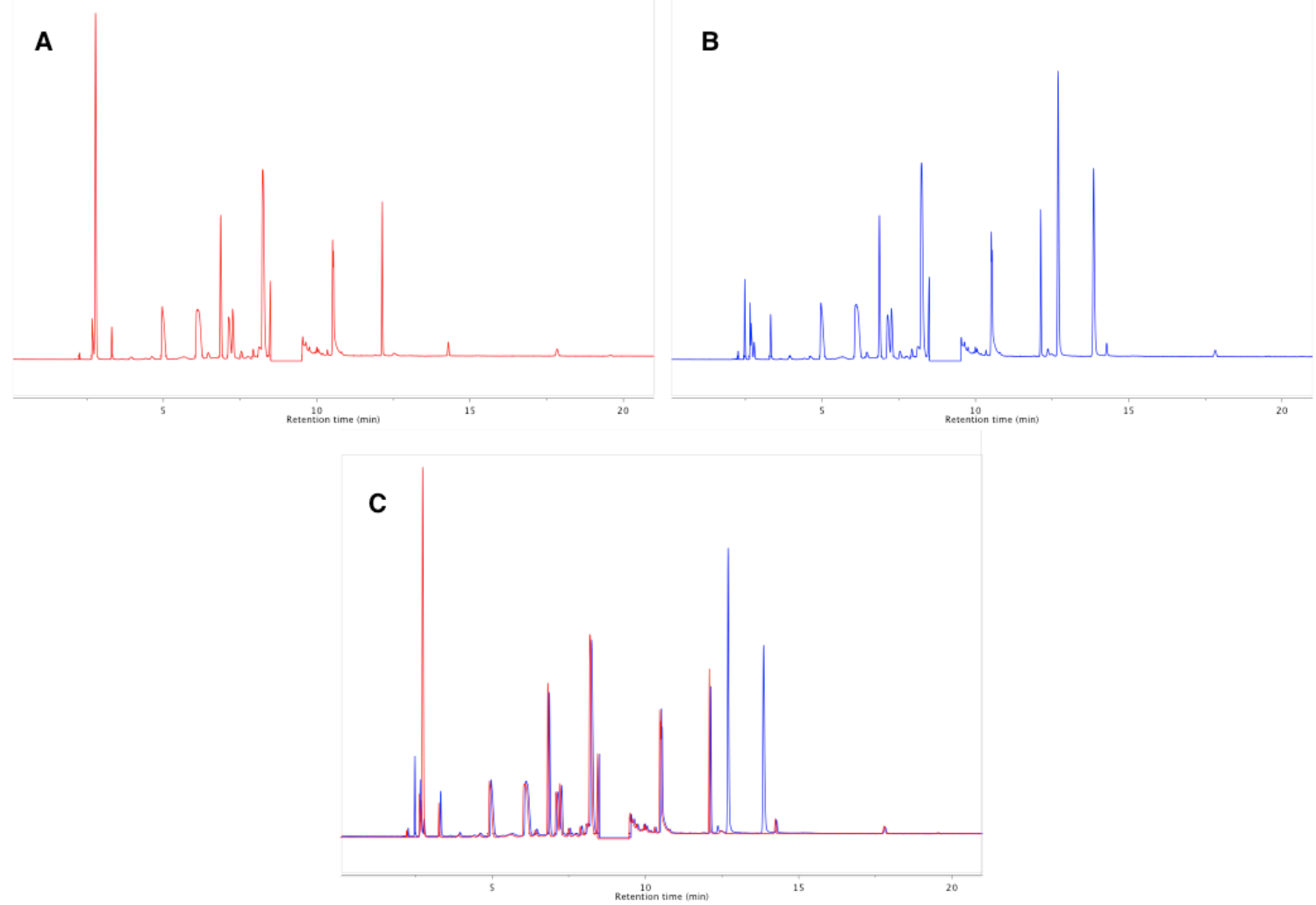

Figure S5. A) GC trace of pure octanol (red). B) GC trace of $45 \mathrm{mM} \delta$-IEPOX (3) dissolved in octanol (blue). C) Overlay of octanol trace (red) with $\delta$-IEPOX (3)/octanol trace (blue). $\delta$-IEPOX diastereomers eluted at 13.86 and 12.70 minutes. Integration ratio of chromatogram peaks at 13.86 and 12.70 reveal match to integration ratio calculated for diastereomers of $\delta$-IEPOX by NMR. 

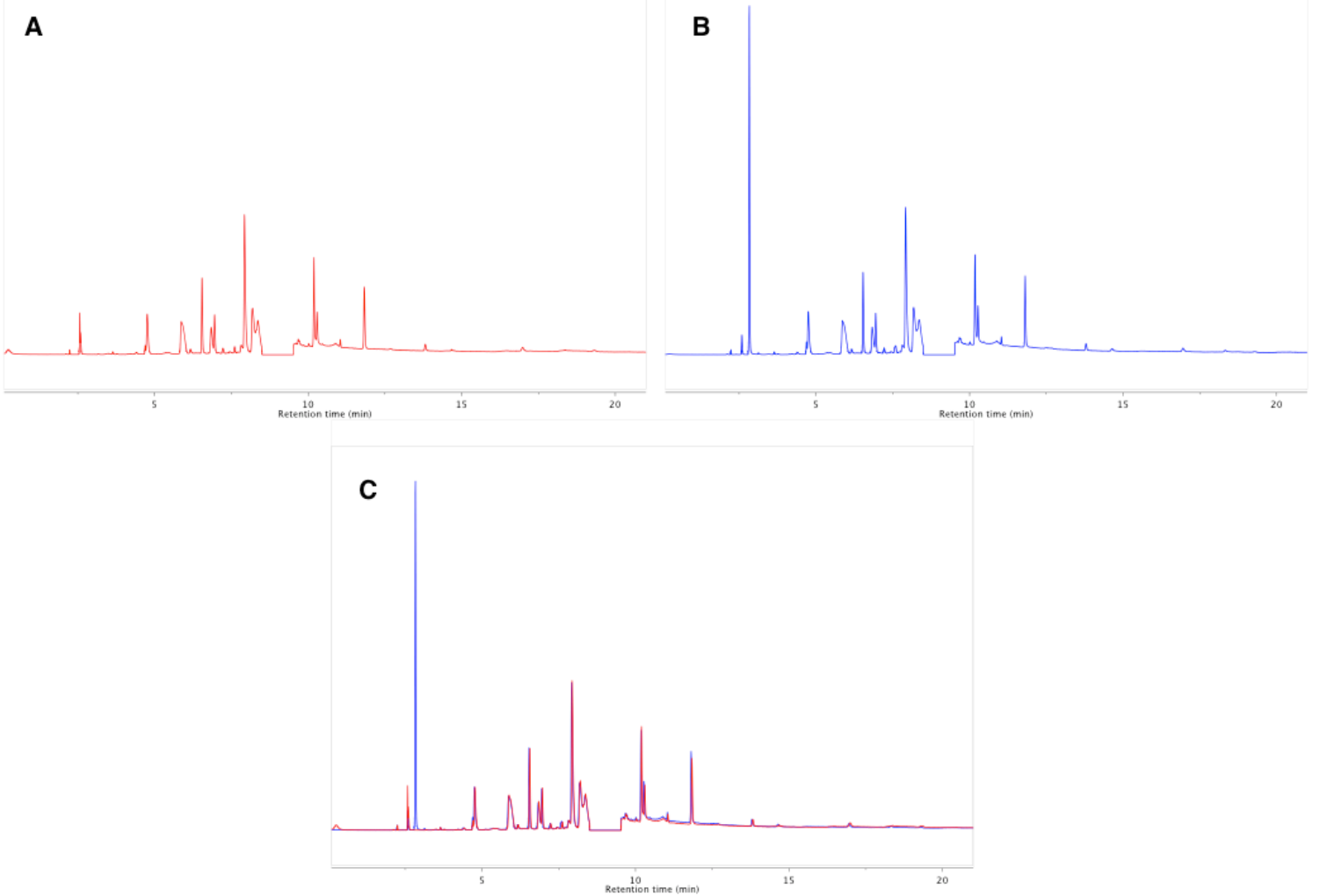

Figure S6. A) GC trace of pure octanol (red). B) GC trace of proposed decomposition product of IEPOX compounds, methyl vinyl ketone (MVK), dissolved in octanol (blue). C) Overlay of octanol trace (red) with MVK/octanol trace (blue). MVK eluted at 2.83 minutes and does not appear to be present at significant concentrations in IEPOX chromatograms. 


\section{REFERENCES}

Herrmann, H., personal communication 4/29/2014

Lin, Y.-H., Zhang, H., Pye, H. O. T., Zhang, Z., Marth, W. J., Park, S., ... Surratt, J. D. (2013). Epoxide as a precursor to secondary organic aerosol formation from isoprene photooxidation in the presence of nitrogen oxides. Proceedings of the National Academy of Sciences of the United States of America, 110(17), 6718-23. doi:10.1073/pnas.1221150110

McNeill, V. F., Woo, J. L., Kim, D. D., Schwier, A. N., Wannell, N. J., Sumner, A. J., \& Barakat, J. M. (2012). Aqueous-phase secondary organic aerosol and organosulfate formation in atmospheric aerosols: a modeling study. Environmental Science \& Technology, 46(15), 8075-81. doi:10.1021/es3002986

Nguyen, T. B., Coggon, M. M., Bates, K. H., Zhang, X., Schwantes, R. H., Schilling, K. A., ... Seinfeld, J. H. (2014). Organic aerosol formation from the reactive uptake of isoprene epoxydiols (IEPOX) onto non-acidified inorganic seeds. Atmospheric Chemistry and Physics, 14(7), 3497-3510. doi:10.5194/acp-14-3497-2014

Sumner, A. J., Woo, J. L., \& McNeill, V. F. (2014). Model analysis of aerosol reaction chamber studies of glyoxal SOA formation: The case for photoenhanced chemistry. Submitted.

Xie, Y., Paulot, F., Carter, W. P. L., Nolte, C. G., Luecken, D. J., Hutzell, W. T., ... Pinder, R. W. (2013). Understanding the impact of recent advances in isoprene photooxidation on simulations of regional air quality. Atmospheric Chemistry and Physics, 13(16), 8439-8455. doi:10.5194/acp-13-8439-2013 